\title{
ON MULTIPLIERS OF $p$-INTEGRABLE FUNCTIONS $\left({ }^{1}\right)$
}

\author{
BY \\ LIANG-SHIN HAHN
}

Introduction. Let $G$ and $\hat{G}$ be two locally compact abelian groups, dual in the sense of Pontrjagin. Let $L^{p}(G), 1 \leqq p \leqq \infty$, be the Banach space of equivalence classes of $p$ th power Haar integrable functions with the usual norm.

Let $\Im: L^{2}(G) \rightarrow L^{2}(\hat{G})$ and $\mathfrak{\Im}: L^{2}(\hat{G}) \rightarrow L^{2}(G)$ be Fourier transform mappings. Haar measures are chosen so that $\Im$ and $\mathfrak{\Im}$ are isometries.

Let $S(G)$ be the space of simple functions on $G$; i.e., finite linear combinations of characteristic functions of measurable sets having compact supports; and $S(\hat{G})$ the corresponding space on $\hat{G}$.

Let $f$ be a bounded measurable function defined on $\hat{G}$. We set

$$
\|f\|_{M_{p}}=\sup \left\{\|\Im f \Im \phi\|_{p} /\|\phi\|_{p} ; \phi \in S(G), \phi \not \equiv 0\right\} .
$$

If $\|f\|_{M_{p}}<\infty$, then the mapping

$$
T_{f}: \phi \rightarrow \widetilde{\Im} f \Im \phi, \phi \in S(G)
$$

has a unique extension to all $L^{p}(G)$, and $f$ will be called a $p$-multiplier.

In the present paper we investigate some sufficient conditions for a function to be a p-multiplier. The history of the problem goes back to Bernstein, Hardy, and Weyl, and has been extensively studied since then, by Calderón, Hirschman, Stein, Zygmund, and many others. The significance of the problem lies in the fact that many classical works in the Fourier analysis can be stated in terms of multipliers. For example, the famous conjugate function theorem of $\mathrm{M}$. Riesz is equivalent to the assertion that $\{i \operatorname{sign} n\}$ is a $p$-multiplier for $1<p<\infty$.

The algebra of $p$-multipliers on $\hat{G}$ will be denoted by $M_{p}(\hat{G})$, or simply by $M_{p}$. Suppose $1 / p+1 / q=1$, and $L^{p} * L^{q}=\left\{f * g ; f \in L^{p}, g \in L^{q}\right\}$, where $f * g$ is the convolution of $f$ and $g$. It is well known (Rudin [11, 1.1.6]) that

$$
L^{p} * L^{q} \subset C_{0}
$$

if $1<p<\infty$.

Since the Fourier transform maps $L^{2}(G)$ onto $L^{2}(\hat{G})$ isometrically, $M_{2}$ consists of precisely the bounded measurable functions; and functions in $L^{1} * L^{\infty}$ are bounded and uniformly continuous (Rudin $[11,1.1 .6]$ ), we have

$$
L^{1} * L^{\infty} \subset M_{2}
$$

Received by the editors August 2, 1966.

(1) To the memory of my father, Shyr-chuan Hahn, M.D. 
Moreover, since $L^{2}(\hat{G}) * L^{2}(\hat{G})$ consists of Fourier transforms of $L^{1}$-functions on $G$ (Rudin $[11,1.6 .3]$ ), we have

$$
L^{2} * L^{2} \subset M_{1}
$$

Thus one is led to investigate whether

$$
L^{p} * L^{q} \subset M_{r}
$$

holds for some other $p, q$, and $r$.

An application of the multilinear version of the Riesz-Thorin interpolation theorem gives our main theorem, which shows that this is the case, and gives a relation between $p, q$, and $r$.

This theorem, together with Calderón's work [3], gives some conditions for a sufficiently smooth function to be in an appropriate $M_{r}$. For example, a function $f$ satisfying an integral Lipschitz condition, say, $f \in \operatorname{Lip}(\alpha, p ; \hat{G})$ for $\alpha>n / p$, $p>2$, where $\hat{G}$ is either a Euclidean space $\mathbf{R}^{n}$ or an $n$-dimensional torus $\mathbf{T}^{n}$, is an $r$-multiplier for $2 p /(p+2) \leqq r \leqq 2 p /(p-2)$.

This proposition, naïve as it appears, enables us to generalize some of the theorems due to Hirschman [7]. For example, combining with the interpolation theorem for Lipschitz spaces, one obtains a generalization of Theorem $4 \mathrm{c}$ in Hirschman [7]. And if combined with the compactness of $\mathbf{T}^{n}$, we have a generalization of Theorem 2a, Hirschman [7]. Furthermore, as a by-product, we have another proof of $L^{p}$ norm convergence $(1<p<\infty)$ for the Bochner-Riesz means below the critical index.

Standard facts in harmonic analysis will be used freely in what follows. Results for which no explicit reference is given will be found in Rudin [11] or Loomis [9].

Acknowledgment. I would like to take this opportunity to express my heartfelt gratitude to Professor Karel de Leeuw for his constant encouragement and guidance throughout my graduate work.

Main theorem. By definition, a bounded measurable function $f$ is in $M_{p}(\hat{G})$ if and only if there is a constant $K$ such that

$$
\|\tilde{\Im} f \Im \phi\|_{p} \leqq K\|\phi\|_{p}
$$

holds for all $\phi \in S(G)$. The infimum of such constants $K$ will be denoted by $\|f\|_{M_{p}}$. Note that $\|\cdot\|_{M_{p}}$ is only a seminorm and not a norm.

LEMMA 1. Let $1<p<\infty$, and $f$ be a bounded measurable function on $\hat{G}$. Then the following are equivalent:

(i) $f \in M_{p}(\hat{G})$;

(ii) There is a constant $K$ such that

$$
\left|\int_{G} f \cdot \Im \Phi \cdot \Im \psi\right| \leqq K\|\Phi\|_{p} \cdot\|\psi\|_{q}
$$

for all $\Phi$ and $\psi$ in $S(G)(1 / p+1 / q=1)$. 
Proof. (i) $\Rightarrow$ (ii). If $f \in M_{p}(\hat{G})$, then by the previous remark,

$$
\|f\|_{M_{p}}=\sup \left\{\|\Im f \Im \Phi\|_{p} ; \Phi \in S(G),\|\Phi\|_{p} \leqq 1\right\} .
$$

But, since $L^{q}(G)$ is the dual of $L^{p}(G)$, and $S(G)$ is dense in $L^{q}(G)$, this is the same as the supremum of

$$
\left\{\left|\int_{G}(\widetilde{\Im} f \Im \Phi) \psi\right| ; \Phi, \psi \in S(G),\|\Phi\|_{p} \leqq 1,\|\psi\|_{q} \leqq 1\right\} .
$$

By Plancherel's theorem, this in turn is the same as the supremum of

$$
\left\{\left|\int_{\hat{G}} f \cdot \Im \Phi \cdot \Im \psi\right| ; \Phi, \psi \in S(G),\|\Phi\|_{p} \leqq 1,\|\psi\|_{q} \leqq 1\right\} .
$$

(ii) $\Rightarrow$ (i). If (ii) holds, reversing the preceding argument one sees that the operator

defined by

$$
T: S(G) \rightarrow L^{p}(G)
$$

$$
T \Phi=\tilde{\Im} f \Im \Phi, \Phi \in S(G),
$$

has $L^{p}(G)$ norm less than or equal to $K$. Thus $T$ extends by continuity to all of $L^{p}(G)$ with the same norm, showing that $f \in M_{p}(\hat{G})$ and $\|f\|_{M_{p}} \leqq K$.

Note that this lemma can easily be extended to multipliers from $L^{p}$ to $L^{q}$. Namely, $f$ is called a $(p, q)$-multiplier if

$$
\sup \left\{\|\tilde{\Im} f \Im \phi\|_{q} /\|\phi\|_{p} ; \phi \in S(G), \phi \not \equiv 0\right\}
$$

is finite. The set of all $(p, q)$-multipliers will be denoted by $M_{p}^{q}(\hat{G})$. Then we have

LEMMA $1^{\prime}$. Let $1 / q+1 / p^{\prime}=1 / q+1 / q^{\prime}=1$. The following are equivalent:

(i) $f \in M_{p}^{q}(\hat{G})$;

(ii) There is a constant $K$ such that

$$
\left|\int_{\hat{G}} f \cdot \Im \Phi \cdot \Im \psi\right| \leqq K\|\Phi\|_{p} \cdot\|\psi\|_{q^{\prime}}
$$

for all $\Phi$ and $\psi$ in $S(G)$.

Note also that $M_{p}^{q}(\hat{G})$ contains only the zero function if $p>q$ and $\hat{G}$ is not compact (Hörmander [8, Theorem 1.1]).

We need two more well-known results (see Hörmander [8, Theorem 1.3]):

Lemma 2. Let $1 / p+1 / p^{\prime}=1 / q+1 / q^{\prime}=1$. Then

in particular,

$$
M_{p}^{q}=M_{q^{\prime}}^{p^{\prime}} ;
$$

$$
M_{p}=M_{p^{\prime}}
$$

LEMMA 3. If $1 \leqq p \leqq q \leqq 2$, then

$$
M_{p} \subset M_{q}
$$


Lemma 2 is a consequence of the duality of $L^{p}$ and $L^{p^{\prime}}$, and Lemma 3 follows from the Riesz-Thorin interpolation theorem (see Zygmund [15, XII, Theorem 1.11]). These two lemmas together assert that the set of points $1 / p$ for which $f \in M_{p}$ is a line segment symmetric with respect to the point $1 / 2$.

Before we proceed to our main theorem, let us make the following

Convention 4. In the sequel, by " $\{\cdots\} \subset M_{r}(\hat{G})$ for $1 \leqq r \leqq \infty "$, we mean: $\{\cdots\} \subset M_{r}(\hat{G})$ for $1 \leqq r \leqq \infty$ if $\hat{G}$ is not compact, and $\{\cdots\} \subset M_{r}(\hat{G})$ for $1 \leqq r \leqq \infty$ if $\hat{G}$ is compact.

This is due to the fact that $S(G)$ is not dense in $L^{\infty}(\hat{G})$ unless $\hat{G}$ is compact.

THEOREM 5. Let $1<p<2,1 / p+1 / q=1$. Then

$$
L^{p}(\hat{G}) * L^{q}(\hat{G}) \subset M_{r}(\hat{G})
$$

for $2 p /(3 p-2) \leqq r \leqq 2 p /(2-p)$; or equivalently, for $2 q /(q+2) \leqq r \leqq 2 q /(q-2)$.

Note that for $p=1$ and $p=2$, Theorem 5 reduces to the trivial facts

$$
L^{1} * L^{\infty} \subset M_{2}
$$

and

$$
L^{2} * L^{2} \subset M_{r} \quad \text { for } 1 \leqq r \leqq \infty .
$$

Proof of the theorem. First, let us prove the following inequality:

$$
\left|\int_{G}(f * g) \cdot \Im \Phi \cdot \Im \psi\right| \leqq\|f\|_{1} \cdot\|g\|_{\infty} \cdot\|\Phi\|_{2} \cdot\|\psi\|_{2}
$$

for all $\Phi$ and $\psi$ in $S(G), f$ and $g$ in $S(\hat{G})$. For,

$$
\begin{aligned}
\left|\int_{\hat{G}}(f * g) \cdot \Im \Phi \cdot \Im \psi\right| & \leqq\|f * g\|_{\infty} \cdot\left|\int_{\hat{G}} \Im \Phi \cdot \Im \psi\right| \\
& \leqq\|f\|_{1} \cdot\|g\|_{\infty} \cdot\|\Im \Phi\|_{2} \cdot\|\Im \psi\|_{2}
\end{aligned}
$$

(by the Schwarz inequality)

$$
\leqq\|f\|_{1} \cdot\|g\|_{\infty} \cdot\|\Phi\|_{2} \cdot\|\psi\|_{2}
$$

(by Plancherel's theorem).

Similarly,

$$
\left|\int_{G}(f * g) \cdot \Im \Phi \cdot \Im \psi\right| \leqq\|f\|_{2} \cdot\|g\|_{2} \cdot\|\Phi\|_{1} \cdot\|\psi\|_{\infty}
$$

holds for all $\Phi$ and $\psi$ in $S(G), f$ and $g$ in $S(\hat{G})$.

Consider the multilinear mapping

$$
T: S(\hat{G}) \times S(\hat{G}) \times S(G) \times S(G) \rightarrow \mathrm{C}
$$

(where $\mathbf{C}$ is the complex field), defined by

$$
T(f, g, \Phi, \psi)=\int_{A}(f * g) \cdot \Im \Phi \cdot \Im \psi .
$$


Inequalities (1) and (2) assert that $T$ satisfies the inequalities

$$
|T(f, g, \Phi, \psi)| \leqq\|f\|_{p} \cdot\|g\|_{q} \cdot\|\Phi\|_{r} \cdot\|\psi\|_{s}
$$

for $p=1, q=\infty, r=2$, and $s=2$; and for $p=2, q=2, r=1$, and $s=\infty$.

By the multilinear version of the Riesz-Thorin interpolation theorem (see Zygmund [15, XII, Theorem 3.3]), $T$ satisfies these inequalities for

$$
\begin{array}{ll}
1 / p=(1-\lambda)\left(\frac{1}{1}\right)+\lambda\left(\frac{1}{2}\right), & 1 / p+1 / q=1, \\
1 / r=(1-\lambda)\left(\frac{1}{2}\right)+\lambda\left(\frac{1}{1}\right), & 1 / r+1 / s=1,
\end{array}
$$

where $0 \leqq \lambda \leqq 1$. Eliminating $\lambda$, we have $1 / p+1 / r=3 / 2$.

This, together with Lemma 1 and the density of $S(\hat{G})$ in $L^{p}(\hat{G})$ and $L^{q}(\hat{G})$, proves that

$$
L^{p}(\hat{G}) * L^{q}(\hat{G}) \subset M_{r}(\hat{G})
$$

for $1 / r=3 / 2-1 / p=(3 p-2) / 2 p$.

Now, applying Lemmas 2 and 3, we have the desired result.

The conclusion of the theorem cannot be strengthened, since as a consequence we will derive a generalization of Theorem 2a of Hirschman [7], whose conclusion is known not to be improvable.

Note that

$$
\|f * g\|_{M_{r}} \leqq\|f\|_{p} \cdot\|g\|_{q}, \quad f \in L^{p}, g \in L^{q} .
$$

Applications $\hat{G}=\mathbf{R}^{n}$. In this section, we will find some applications of the main theorem to $n$-dimensional Euclidean space $\mathbf{R}^{n}$.

For $x=\left(x_{1}, \ldots, x_{n}\right) \in \mathbf{R}^{n}$, set $|x|=\left[\sum_{j=1}^{n} x_{j}^{2}\right]^{1 / 2}$.

From now on, the Fourier transform of a function $f$ will be denoted by $f^{\wedge}$.

Definition 6. Let $\alpha$ be a complex number and $1 \leqq p \leqq \infty$. We define $L_{\alpha}^{p}\left(\mathbf{R}^{n}\right)$ to be the image of $L^{p}\left(\mathbf{R}^{n}\right)$ under the so-called Bessel potential operator $J_{\alpha}$ of order $\alpha$, where $J_{\alpha}$ is defined by

$$
\left(J_{\alpha} f\right)^{\wedge}=\left(1+4 \pi^{2}|x|^{2}\right)^{-\alpha / 2} f^{\wedge} .
$$

This makes sense for $\operatorname{Re}(\alpha)>0$, and the operators $J_{\alpha}$ form a semigroup.

For the sake of understanding the operator $J_{\alpha}$ and the space $L_{\alpha}^{p}$, we state the following theorem; the proof of it can be found in Calderón [3, Theorems 1 and 2]:

THEOREM 7. For $\operatorname{Re}(\alpha)>0$ and $1 \leqq p \leqq \infty$, the operator $J_{\alpha}$ transforms $L^{p}\left(\mathbf{R}^{n}\right)$ continuously into itself, with norm less than or equal to 1.

If $\operatorname{Re}(\alpha) \geqq|k|$, then $(\partial / \partial x)^{k} J_{\alpha}$ transforms $L^{p}\left(\mathbf{R}^{n}\right)$ continuously into itself.

Thus it is reasonable to think that the space $L_{\alpha}^{p}, 1<p<\infty$, with $\alpha$ a positive integer, coincides with the space of functions which together with their derivatives up to and including $\alpha$ belong to $L^{p}$. 
Hereafter $\alpha$ will be a positive real number. We need the following results (Calderón [3, Theorems 5 and 6]):

THEOREM 8. (a) If $\alpha \leqq \beta$, then $L_{\alpha}^{p} \supset L_{\beta}^{p}$.

(b) Let $p>1, \alpha \geqq \beta$, and $1 / q=1 / p-(\alpha-\beta) / n>0$; then $L_{\alpha}^{p} \subset L_{\beta}^{p}$.

For $0<\alpha<n$, the function $\left(1+4 \pi^{2}|x|^{2}\right)^{-\alpha / 2}$ is the Fourier transform of an integrable function $K_{\alpha}(x)$, where

$$
K_{\alpha}(x)=\gamma(\alpha) e^{-|x|} \int_{0}^{\infty} e^{-|x| t}\left(t+\frac{t^{2}}{2}\right)^{(n-\alpha-1) / 2} d t
$$

with

$$
\gamma^{-1}(\alpha)=(2 \pi)^{(n-1) / 2} \Gamma\left(\frac{\alpha}{2}\right) \Gamma\left(\frac{n-\alpha+1}{2}\right)
$$

(see Aronszajn and Smith [1]).

Thus, by redefining on a set of measure zero, $L_{\alpha}^{p}$ is the space of functions $f$ of the form

$$
f=J_{\alpha} \Phi=K_{\alpha} * \Phi, \quad \Phi \in L^{p}\left(\mathbf{R}^{n}\right) .
$$

Suppose $0<\alpha<n$, then for $|x| \geqq 1$, the integral on the right of (3) is bounded, and thus the right-hand side is $O\left(e^{-|x|}\right)$. For $|x| \leqq 1$, the integral is dominated by

$$
C\left[1+\int_{1}^{\infty} e^{-|x| t} t^{n-\alpha-1} d t\right] \leqq C|x|^{-n+\alpha}
$$

Hence

$$
\left|K_{\alpha}(x)\right| \leqq C_{\alpha} e^{-|x|} \cdot|x|^{-n+\alpha}
$$

From this we get

$$
K_{\alpha} \in L^{q}\left(\mathbf{R}^{n}\right) \text { if } n / p<\alpha<n, \quad 1 / p+1 / q=1 .
$$

THEOREM 9. (a) If $1<p \leqq 2$ and $\alpha>n / p$, then $L_{\alpha}^{p}\left(\mathbf{R}^{n}\right) \subset M_{r}\left(\mathbf{R}^{n}\right)$ for $1 \leqq r<\infty$.

(b) If $q>2$ and $\alpha>n / q$, then $L_{\alpha}^{q}\left(\mathbf{R}^{n}\right) \subset M_{r}\left(\mathbf{R}^{n}\right)$ for $2 q /(q+2) \leqq r \leqq 2 q /(q-2)$.

Proof. By Theorem 8(a), $L_{\alpha}^{p} \subset L_{\beta}^{p}$ if $\alpha \geqq \beta$, so we may consider the case $n / p<\alpha<n$ only.

As $L_{\alpha}^{p}$ is the class of functions $f$ on the form

$$
f=J_{\alpha}(\Phi)=K_{\alpha} * \Phi,
$$

where $\Phi \in L^{p}$, we have, by (4)

$$
L_{\alpha}^{p} \subset L^{p} * L^{q}, \quad 1 / p+1 / q=1 .
$$

Hence from Theorem 5 , we get the part (b); and for the case $1<p \leqq 2$,

$$
L_{\alpha}^{p} \subset M_{r} \quad \text { for } 2 p /(3 p-2) \leqq r \leqq 2 p /(2-p) .
$$


In particular, if $\beta>n / 2, L_{\beta}^{2} \subset M_{r}$ for $1 \leqq r<\infty$. Now, by Theorem 8(b),

$$
L_{\alpha}^{p} \subset L_{\beta}^{2} \quad \text { if } \frac{1}{2}=1 / p-(\alpha-\beta) / n,
$$

and since $\beta-n / 2=\alpha-n / p>0$, the proof is complete.

REMARK 10. The conclusion is still valid even if $p=1$; simply pick $\beta$ such that $n / 2<\alpha-\beta, n / 2<\beta<\alpha-n / 2$, and proceed as before. (See Calderón [3, Remark after Theorem 6].)

Definition 11. Let $\alpha$ be a real number and $\alpha_{*}$ the largest integer less than $\alpha$. Let $\Lambda_{\alpha}^{p}=\Lambda_{\alpha}^{p}\left(\mathbf{R}^{n}\right)$ be the class of functions $f, f \in L_{\alpha_{*}}^{p}$ such that

$$
\left\|\Delta_{h}^{2} J_{-\alpha_{*}} f\right\|_{p} \leqq O\left(|h|^{\alpha-\alpha_{*}}\right), \quad h \in \mathbf{R}^{n},
$$

where $\Delta_{h} f(x)=f(x+h)-f(x)$.

Also let $\operatorname{Lip}(\alpha, p)=\operatorname{Lip}\left(\alpha, p ; \mathbf{R}^{n}\right)$ be the class of functions $f$ such that for $|k|<\alpha_{*},(\partial / \partial x)^{k} f \in L^{p}$, and for $|k|=\alpha_{*}$,

$$
\begin{cases}\left\|\Delta_{h}\left(\frac{\partial}{\partial x}\right)^{k} f\right\|_{p}=O\left(|h|^{\alpha-\alpha_{*}}\right) & \text { if } \alpha-\alpha_{*}<1 ; \\ \left\|\Delta_{h}^{2}\left(\frac{\partial}{\partial x}\right)^{k} f\right\|_{p}=O(|h|) & \text { if } \alpha-\alpha_{*}=1 .\end{cases}
$$

The following fact is known (Calderón [3, Theorem 8]):

THEOREM 11. (a) If $\alpha$ is not an integer,

$$
\Lambda_{\alpha}^{p}=\operatorname{Lip}(\alpha, p) .
$$

(b) If $1<p<\infty$, then $J_{\beta}$ maps $\Lambda_{\alpha}^{p}$ onto $\Lambda_{\alpha+\beta}^{p}$.

(c) If $1<p<\infty$ and $\alpha>\beta$, then

$$
\Lambda_{\alpha}^{p} \subset L_{\beta}^{p} \subset \Lambda_{\beta}^{p} .
$$

With these preliminaries we now prove the theorem announced in the introduction.

THEOREM 12. (a) If $1<p \leqq 2, \alpha>n / p$, then $\operatorname{Lip}\left(\alpha, p ; \mathbf{R}^{n}\right) \subset M_{r}\left(\mathbf{R}^{n}\right)$ for $1 \leqq r<\infty$.

(b) If $q>2, \alpha>n / q$, then $\operatorname{Lip}\left(\alpha, q ; \mathbf{R}^{n}\right) \subset M_{r}\left(\mathbf{R}^{n}\right)$ for $2 q /(q+2) \leqq r \leqq 2 q /(q-2)$.

Proof. If $\alpha$ is not an integer, pick $\beta$ such that $\alpha>\beta>n / p$, and applying Theorem 11 , we have

$$
\operatorname{Lip}(\alpha, p)=\Lambda_{\alpha}^{p} \subset L_{\beta}^{p} .
$$

Now Theorem 9 gives the desired result for this case.

If $\alpha$ is an integer, let $\beta$ be a noninteger and $\alpha>\beta>n / p$, then

$$
\operatorname{Lip}(\alpha, p) \subset \operatorname{Lip}(\beta, p) .
$$

Now the previous paragraph applies to $\operatorname{Lip}(\beta, p)$, and we get the desired result again. 
We need the following very special case of the interpolation theorem for Lipschitz spaces obtained by Taibleson [13, Theorem 8]:

THEOREM 13. Suppose $f \in \operatorname{Lip}\left(\alpha_{i}, p_{i}\right), i=0,1$, and let $\alpha=(1-\lambda) \alpha_{0}+\lambda \alpha_{1}$, $1 / p=(1-\lambda) / p_{0}+\lambda / p_{1}$, where $0 \leqq \lambda \leqq 1$. Then

$$
f \in \operatorname{Lip}(\alpha, p) \text {. }
$$

THEOREM 14. Let $0<\alpha \leqq n / p, \beta>n / q$, and $2 \leqq p<q$. Then

$$
\operatorname{Lip}\left(\alpha, p ; \mathbf{R}^{n}\right) \cap \operatorname{Lip}\left(\beta, q ; \mathbf{R}^{n}\right) \subset M_{r}\left(\mathbf{R}^{n}\right)
$$

for $2[1+2((1-\mu) / p+\mu / q)]^{-1}<r<2[1-2((1-\mu) / p+\mu / q)]^{-1}$, where $\mu$ satisfies $(1-\mu) \alpha+\mu \beta=n((1-\mu) / p+\mu / q)$.

Proof. From Theorem 13, we have

$$
\operatorname{Lip}(\alpha, p) \cap \operatorname{Lip}(\beta, q) \subset \operatorname{Lip}(\tau, t)
$$

for $\tau=(1-\lambda) \alpha+\lambda \beta, 1 / t=(1-\lambda) / p+\lambda / q, 0 \leqq \lambda \leqq 1$. If $\mu<\lambda \leqq 1$, then $\tau>n / t$, hence

$$
\operatorname{Lip}(\tau ; t) \subset M_{r} \text { for } 2(1+2 / t)^{-1} \leqq r \leqq 2(1-2 / t)^{-1},
$$

by Theorem 12, and the desired result follows.

Remark 15. The case $p=2, q=\infty$, was obtained by Hirschman [7, Theorem 4c].

REMARK 16. The condition $2 \leqq p<q$ is not essential. Even if $p<2<q$, we can still improve the result obtainable from Theorem 12 by the same argument.

As an application of Theorem 14, we prove that the Bochner-Riesz means for multiple Fourier integrals converges in $L^{p}$ norm $(1<p<\infty)$ below the critical index $\delta=(n-1) / 2$.

Let $1<p \leqq 2, \delta>(2 / p-1)(n-1) / 2$ and

$$
\begin{aligned}
S_{\delta, R}(x) & =\left[1-(|x| / R)^{2}\right]^{\delta}, & & |x|<R ; \\
& =0, & & |x| \geqq R .
\end{aligned}
$$

Then by computation

$$
S_{\delta, R} \in \operatorname{Lip}(\delta, \infty) \cap \operatorname{Lip}(\delta+1 / 2,2) .
$$

Hence from Theorem 14, $S_{\delta, R} \in M_{p}$.

Let

$$
S_{\delta, R}(f ; x)=\int_{|y|<R}\left[1-(|y| \mid R)^{2}\right]^{\delta} e^{2 \pi i<x, y\rangle} f^{\wedge}(y) d y .
$$

By the similarity argument, $S_{\delta, R}$ as an operator on $L^{p}\left(\mathbf{R}^{n}\right)$, has a norm independent of $R$. Hence by the uniform.boundedness theorem,

$$
S_{\delta, R}(f) \rightarrow f
$$

in $L^{p}$ as $R \rightarrow \infty$. (See Stein [12].) 
Definition 17. Let $f$ be a function defined on $\mathbf{R}$. Let

$$
V_{p}[f]=\sup \left\{\sum_{0}^{n-1}\left|f\left(x_{k+1}\right)-f\left(x_{k}\right)\right|^{p}\right\}^{1 / p},
$$

where the supremum is taken over all sets of real numbers $x_{0}<x_{1}<\cdots<x_{n}$. $V_{p}[f]$ will be called the $p$-variation of $f$.

THEOREM 18. If $f$ is a function defined on $\mathbf{R}$, and

1. $f \in L^{p}(\mathbf{R}), p \geqq 2$;

2. $V_{p}[f]<\infty$;

3. $|f(x+h)-f(x)| \leqq O\left(|h|^{\circ}\right)$ for some $\delta>0$.

Then $f \in M_{r}$ for $2 p /(p+2)<r<2 p /(p-2)$.

Proof. Let us consider

$$
\left\|\Delta_{h} f\right\|_{p}^{p}=\int_{\mathbf{R}}|f(x+h)-f(x)|^{p} d x .
$$

Without loss of generality, we may assume that $h>0$. Then

$$
\left\|\Delta_{h} f\right\|_{p}^{p}=\sum_{k \in \mathbf{Z}} \int_{k h}^{(k+1) h}|f(x+h)-f(x)|^{p} d x,
$$

where $\mathbf{Z}$ is the group of integers.

Let $x_{k}$ be that value of $x$ in $[k h,(k+1) h]$ for which $|f(x+h)-f(x)|$ is a maximum. Then

$$
\left\|\Delta_{h} f\right\|_{p}^{p} \leqq h\left[\sum_{1}+\sum_{2}\right]
$$

where

$$
\sum_{1}=\sum_{k \text { odd }}\left|f\left(x_{k}+h\right)-f\left(x_{k}\right)\right|^{p}
$$

and

$$
\sum_{2}=\sum_{k \text { even }}\left|f\left(x_{k}+h\right)-f\left(x_{k}\right)\right|^{p} .
$$

Since $x_{k}<x_{k}+h<x_{k+2}$ for all $k \in \mathbf{Z}$, we see that $\sum_{1}$ and $\sum_{2}$ are both not greater than $V_{p}^{p}[f]$, and thus

$$
\left\|\Delta_{h} f\right\|_{p}^{p} \leqq 2 h V_{p}^{p}[f] .
$$

And since we have assumed that $f \in L^{p}(\mathbf{R})$, we get

$$
f \in \operatorname{Lip}(1 / p, p) \text {. }
$$

By assumption $3, f$ also belongs to $\operatorname{Lip}(\delta, \infty)$. Thus by Theorem 14 , we have the desired result.

Note that Theorem 18 contains Theorem 4e of Hirschman [7] for the case $\delta>1 / p$. 
Note also that the condition $p \geqq 2$ can be changed to $1 \leqq p \leqq 2$; in that case we have

$$
f \in M_{r} \quad \text { for } 1 \leqq r<\infty .
$$

Applications $\hat{G}=\mathbf{T}^{n}$. Now let us consider the $n$-dimensional torus $\mathbf{T}^{n}=(-1 / 2,1 / 2]^{n}$. Suppose $\alpha>0$, and define

$$
K_{\alpha}^{*}(\theta)=\sum_{k} K_{\alpha}(\theta+k), \quad \theta \in \mathbf{T}^{n},
$$

where the summation runs through all lattice points $k=\left(k_{1}, k_{2}, \ldots, k_{n}\right)$ in $\mathbf{R}^{n}$.

From our former estimate

$$
\left|K_{\alpha}(x)\right| \leqq C_{\alpha} e^{-|x| / 2}|x|^{-n+\alpha},
$$

the sum in (5) converges absolutely to a continuous function except at the origin. Furthermore, for $m \in \mathbf{Z}^{n}$,

$$
\begin{aligned}
K_{\alpha}^{* \wedge}(m) & =\int_{\mathbf{T}^{n}} e^{-2 \pi i\langle m, \theta\rangle} K_{\alpha}^{*}(\theta) d \theta \\
& =\int_{\mathbf{T}^{n}} e^{-2 \pi i\langle m, \theta\rangle}\left[\sum_{k \in \mathbf{Z}^{n}} K_{\alpha}(\theta+k)\right] d \theta \\
& =\sum_{k \in \mathbf{Z}^{n}} \int_{\mathbf{T}^{n}} e^{-2 \pi i\langle m, \theta+k\rangle} K_{\alpha}(\theta+k) d \theta \\
& =\int_{\mathbf{R}^{n}} e^{-2 \pi i\langle m, x\rangle} K_{\alpha}(x) d x \\
& =K_{\alpha}^{\wedge}(m) \\
& =\left(1+|m|^{2}\right)^{-\alpha / 2} .
\end{aligned}
$$

And by the inversion formula, we have

$$
K_{\alpha}^{*}(\theta)=\sum_{k \in \mathbf{Z}^{n}}\left(1+|k|^{2}\right)^{-\alpha / 2} e^{2 \pi i\langle k, \theta\rangle} .
$$

As before, we have

$$
K_{\alpha}^{*} \in L^{q}\left(\mathbf{T}^{n}\right) \quad \text { if } \quad \alpha>n / p, \quad 1 / p+1 / q=1 .
$$

And the analogue of Theorem 11 holds if we define $L_{\alpha}^{p}\left(\mathbf{T}^{n}\right), \Lambda_{\alpha}^{p}\left(\mathbf{T}^{n}\right)$ and $\operatorname{Lip}\left(\alpha, p ; \mathbf{T}^{n}\right)$ accordingly. Thus torus version of Theorems 12 and 14 are of course true; namely,

THEOREM $12^{\prime}$. (a) If $1<p \leqq 2, \alpha>n / p$, then $\operatorname{Lip}\left(\alpha, p ; \mathbf{T}^{n}\right) \subset M_{r}\left(\mathbf{T}^{n}\right)$ for $1 \leqq r \leqq \infty$. (b) If $q>2, \alpha>n / q$, then $\operatorname{Lip}\left(\alpha, q ; \mathrm{T}^{n}\right) \subset M_{r}\left(\mathrm{~T}^{n}\right)$ for $2 q /(q+2) \leqq r \leqq 2 q /(q-2)$.

THEOREM $14^{\prime}$. Let $0<\alpha \leqq n / p, \beta>n / q$, and $2 \leqq p<q$. Then

$$
\operatorname{Lip}\left(\alpha, p ; \mathbf{T}^{n}\right) \cap \operatorname{Lip}\left(\beta, q ; \mathbf{T}^{n}\right) \subset M_{r}\left(\mathbf{T}^{n}\right)
$$


for $2[1+2((1-\mu) / p+\mu / q)]^{-1}<r<2[1-2((1-\mu) / p+\mu / q)]^{-1}$, where $\mu$ satisfies $(1-\mu) \alpha+\mu \beta=n((1-\mu) / p+\mu / q)$.

Again the condition $2 \leqq p<q$ is not essential.

As an application of our argument, we prove the following (Zygmund [15, VI, Theorem 3.6]):

THEOREM 19. If $f$ is of bounded variation on $\mathbf{T}$ and satisfies a Lipschitz condition of order $\alpha$, for some $\alpha>0$, then the Fourier series of $f$ converges absolutely.

Proof. Since $f$ is of bounded variation means $f \in \operatorname{Lip}(1,1)$, (see Zygmund [15, Vol. I, p. 180]). Hence

$$
f \in \operatorname{Lip}(\alpha, \infty) \cap \operatorname{Lip}(1,1) .
$$

Thus by the interpolation theorem for Lipschitz spaces,

$$
f \in \operatorname{Lip}\left(\frac{\alpha+1}{2}, 2\right) .
$$

Now from Theorem $12^{\prime}, f \in M_{r}$ for $1 \leqq r \leqq \infty$. Therefore the Fourier series of $f$ converges absolutely. For, $g \in L^{1}(\mathbf{Z})$ implies $f \hat{g}$ is a Fourier transform of a function in $L^{1}(Z)$. In particular, if we take

$$
\begin{aligned}
g(n) & =1, & & n=0 ; \\
& =0, & & n \neq 0,
\end{aligned}
$$

then $\hat{g}(\theta) \equiv 1$ for all $\theta \in \mathbf{T}$. Hence $f$ itself is a Fourier transform of $L^{1}(\mathbf{Z})$-function.

Again, as in the case of Euclidean space, Theorem 14' implies $L^{p}$ norm convergence for Bochner-Riesz mean of multiple Fourier series (see Stein [12]). Let

where

$$
S_{\delta, R}(f, \theta)=\sum_{|k| \leqq R} a_{k}\left[1-(|k| / R)^{2}\right]^{\delta} e^{2 \pi i\langle k, \theta\rangle},
$$

Then

$$
a_{k}=\int_{\mathbf{T}^{n}} e^{-2 \pi i<k, \theta\rangle} f(\theta) d \theta .
$$

$$
S_{\delta, R}(f) \rightarrow f \text { in } L^{p}
$$

whenever $f \in L^{p}$, provided $\delta>(2 / p-1)(n-1) / 2,1<p \leqq 2$, or $\delta>\left(2 / p^{\prime}-1\right)(n-1) / 2$, $2 \leqq p<\infty, 1 / p+1 / p^{\prime}=1$.

Moreover, since $\mathbf{T}^{n}$ is compact, we have

$$
\operatorname{Lip}\left(\alpha, p ; \mathbf{T}^{n}\right) \supset \operatorname{Lip}\left(\alpha, q ; \mathbf{T}^{n}\right) \quad \text { if } p \leqq q .
$$

Combining this with Theorem $12^{\prime}$, we obtain

THEOREM 20. Suppose $n / q<\alpha \leqq n / 2 ;$ then

$$
\operatorname{Lip}\left(\alpha, q ; \mathbf{T}^{n}\right) \subset M_{r}\left(\mathbf{T}^{n}\right)
$$

for $2 n /(n+2 \alpha)<r<2 n /(n-2 \alpha)$. 
Proof. Since

$$
\operatorname{Lip}\left(\alpha, q ; \mathbf{T}^{n}\right) \subset \operatorname{Lip}\left(\alpha, p ; \mathbf{T}^{n}\right) \quad \text { if } p \leqq q,
$$

and

$$
\operatorname{Lip}\left(\alpha, p ; \mathbf{T}^{n}\right) \subset M_{r}\left(\mathbf{T}^{n}\right)
$$

for $2 p /(p+2) \leqq r \leqq 2 p /(p-2)$ if $\alpha>n / p$, we have the desired result.

Remark 21. The case $q=\infty, n=1$ is due to Hirschman [7, Theorem 2a].

Note that if $\alpha>n / 2, q \geqq 2$, then $\operatorname{Lip}\left(\alpha, q ; \mathbf{T}^{n}\right) \subset M_{r}\left(\mathbf{T}^{n}\right)$ for $1 \leqq r \leqq \infty$. This statement contains the $n$-dimensional torus version of Bernstein's theorem (see Zygmund [8, VI, Theorem 3.1]):

THEOREM 22. Every function in $\operatorname{Lip}\left(\alpha, \infty ; \mathbf{T}^{n}\right)$ has absolutely convergent Fourier series if $\alpha>n / 2$.

Note also, by comparing Theorem 20 with Theorem 14', one finds that neither implies the other.

The following is the analogue of Theorem 18 on the circle first obtained by Hirschman [7, Theorem 2e]:

THEOREM 18'. Let $f$ be a function defined on T. If

1. $V_{p}[f]<\infty, p \geqq 2$;

2. $|f(\theta+h)-f(\theta)| \leqq O\left(|h|^{\delta}\right)$, for some $\delta>0$.

Then $f \in M_{r}(\mathbf{T})$ for $2 p /(p+2)<r<2 p /(p-2)$.

Proof. Since $\mathbf{T}$ is compact, condition 2 implies $f \in L^{p}(\mathbf{T})$. The rest of the proof is exactly the same as that for Theorem 18 .

Note again that if the condition $p>2$ is replaced by $1 \leqq p \leqq 2$, we have

$$
f \in M_{r}(\mathbf{T}) \text { for } 1 \leqq r \leqq \infty .
$$

Application $\hat{G}=\mathbf{Z}$. Finally, let us consider the group $\mathbf{Z}$ of integers.

Definition 23. Let $f$ be a function defined on $\mathbf{Z}$. By $f \in \operatorname{Lip}^{*}(\alpha, p ; Z)$, we mean $f \in L^{p}(\mathbf{Z})$ and there exists a constant $K$ such that

$$
\left\|\Delta_{m} f\right\|_{L^{p}(\mathbf{Z})} \leqq K\left(|m|^{\alpha}\right) \text { for all } m \in \mathbf{Z},
$$

where $\Delta_{m} f(x)=f(x+m)-f(x)$.

Note the difference between the $\operatorname{spaces} \operatorname{Lip}(\alpha, p)$ and $\operatorname{Lip} *(\alpha, p)$. The former emphasizes small $|h|, \Delta_{h} f(x)=f(x+h)-f(x)$ (see Zygmund [15, II]), while the latter is defined on a discrete group.

The following includes the analogue of Theorems 18 and $18^{\prime}$ on $\mathbf{Z}$ :

THEOREM 24. Suppose $p>2$ and $0<\alpha<1$. Then

$$
\operatorname{Lip}^{*}(\alpha, p ; \mathbf{Z}) \subset M_{r}(\mathbf{Z}) \text { for } 2 p /(p+2) \leqq r \leqq 2 p /(p-2) \text {. }
$$


Proof. Since

$$
\operatorname{Lip}^{*}(\alpha, p ; \mathbf{Z}) \supset \operatorname{Lip}^{*}(\beta, p ; \mathbf{Z}) \quad \text { if } \alpha \geqq \beta ;
$$

it is sufficient to prove for $1 / p<\alpha<1$.

Let $f \in \operatorname{Lip}^{*}(\alpha, p ; \mathbf{Z})$. Without loss of generality, we may suppose that $f$ is real-valued. Let $g$ be the polygonal function on $\mathbf{R}$ that interpolates $f$ linearly. $\left.g\right|_{\mathbf{z}}=f$. We shall show that

$$
\begin{aligned}
g & \in \operatorname{Lip}(\alpha, p ; \mathbf{R}) . \\
\|g\|_{L^{p}(\mathbf{R})}^{p} & =\int_{\mathbf{R}}|g(x)|^{p} d x \\
& =\sum_{n \in \mathbf{Z}} \int_{n}^{n+1}|g(x)|^{p} d x .
\end{aligned}
$$

Since $|g(x)|^{p}$ is a convex function of $x$ on $[n, n+1]$ for each $n \in \mathbf{Z}$,

$$
\begin{aligned}
\|g\|_{p}^{p} & \leqq \sum_{n \in \mathbf{Z}} \max \left\{|g(n)|^{p},|g(n+1)|^{p}\right\} \\
& =\sum_{n \in \mathbf{Z}} \max \left\{|f(n)|^{p},|f(n+1)|^{p}\right\} \\
& \leqq 2\|f\|_{L^{p}(\mathbf{Z})}^{p^{p}}<\infty .
\end{aligned}
$$

Therefore

$$
g \in L^{p}(\mathbf{R})
$$

Consider

$$
\left\|\Delta_{h} g\right\|_{p}^{p}=\int_{\mathbf{R}}|g(x+h)-g(x)|^{p} d x .
$$

Without loss of generality, we may assume that $h>0$.

Suppose $h \geqq 1$, then

$$
\begin{aligned}
\left\|\Delta_{h} g\right\|_{p}^{p} & =\sum_{n \in \mathbf{Z}} \int_{n}^{n+1}|g(x+h)-g(x)|^{p} d x \\
& =\sum_{n \in \mathbf{Z}}\left[\int_{n}^{n+1+[h]-h}+\int_{n+1+[h]-h}^{n+1}\right] .
\end{aligned}
$$

Since the integrand is convex in each interval, we have

$$
\begin{aligned}
\left\|\Delta_{h} g\right\|_{p}^{p} \leqq & \sum_{n \in \mathbf{Z}}(1+[h]-h) \cdot \max \left\{|f(n+[h])-f(n)|^{p},|f(n+[h]+1)-f(n)|^{p}\right\} \\
& +\sum_{n \in \mathbf{Z}}(h-[h]) \cdot \max \left\{|f(n+[h]+1)-f(n+1)|^{p},|f(n+[h]+2)-f(n+1)|^{p}\right\} \\
\leqq & 2 \max \left\{\left\|\Delta_{[h]} f\right\|_{p}^{p},\left\|\Delta_{[h]+1} f\right\|_{p}^{p}\right\} \\
= & O\left(h^{\alpha p}\right) .
\end{aligned}
$$


If $0<h<1$, then

$$
\begin{aligned}
\left\|\Delta_{h} g\right\|_{p}^{p} & =\sum_{n \in \mathbf{Z}} \int_{n}^{n+1}|g(x+h)-g(x)|^{p} d x \\
& =\sum_{n \in \mathbf{Z}}\left[\int_{n}^{n+1-h}|g(x+h)-g(x)|^{p} d x+\int_{n+1-h}^{n+1}|g(x+h)-g(x)|^{p} d x\right] .
\end{aligned}
$$

Since the integrand is constant in the first interval, and convex in the second, we have

$$
\begin{aligned}
\left\|\Delta_{h} g\right\|_{p}^{p} \leqq & \sum_{n \in \mathbf{Z}}(1-h)|h[g(n+1)-g(n)]|^{p} \\
& +\sum_{n \in \mathbf{Z}} h \cdot \max \left\{|g(n+1)-g(n+1-h)|^{p},|g(n+1+h)-g(n+1)|^{p}\right\} \\
\leqq & \sum_{n \in \mathbf{Z}}(1-h)|h[g(n+1)-g(n)]|^{p} \\
& +\sum_{n \in \mathbf{Z}} h \cdot \max \left\{|h[g(n+1)-g(n)]|^{p},|h[g(n+2)-g(n+1)]|^{p}\right\} \\
\leqq & \sum_{n \in \mathbf{Z}} h^{p}|f(n+1)-f(n)|^{p}+\sum_{n \in \mathbf{Z}} h^{1+p}|f(n+2)-f(n+1)|^{p} \\
= & O\left(h^{p}\right)+O\left(h^{1+p}\right) \\
= & O\left(h^{p}\right) \\
\leqq & O\left(h^{\alpha p}\right) .
\end{aligned}
$$

Hence

$$
g \in \operatorname{Lip}(\alpha, p ; \mathbf{R})
$$

Therefore, by Theorem 12,

$$
g \in M_{r}(\mathbf{R}) \text { for } 2 p /(p+2) \leqq r \leqq 2 p /(p-2),
$$

since we are assuming that $\alpha>1 / p$.

Applying Proposition 3.3 of de Leeuw [4], we have

$$
f=\left.g\right|_{\mathbf{Z}} \in M_{r}(\mathbf{Z}) \text { for } 2 p /(p+2) \leqq r \leqq 2 p /(p-2) .
$$

Theorem 24 contains Theorem $3 b$ of Hirschman [7] for the case $p \delta>1$.

Note if $1<p \leqq 2,0<\alpha<1$, then $\operatorname{Lip}^{*}(\alpha, p ; \mathbf{Z}) \subset M_{r}(\mathbf{Z})$ for $1 \leqq r<\infty$.

The interpolation theorem for $\operatorname{Lip}^{*}(\alpha, p)$ holds, and we have

TheOREM 25. Suppose $\alpha>1>\beta>0$ and $2 \leqq p<q$, then

$$
\operatorname{Lip}^{*}(\alpha, p ; \mathbf{Z}) \cap \operatorname{Lip}^{*}(\beta, q ; \mathbf{Z}) \subset M_{r}(\mathbf{Z})
$$

for $2[1+2((1-\mu) / p+\mu / q)]^{-1}<r<2[1-2((1-\mu) / p+\mu / q)]^{-1}$ where $\mu$ satisfies $(1-\mu) \alpha+\mu \beta=1$. 


\section{REFERENCES}

1. N. Aronszajn and K. T. Smith, Theory of Bessel potentials. I, Ann. Inst. Fourier (Grenoble) 11 (1961), 385-475.

2. S. Bochner, Summation of multiple Fourier series by spherical means, Trans. Amer. Math. Soc. 40 (1936), 175-207.

3. A. P. Calderón, Lebesgue spaces of differentiable functions and distributions, Proc. Sympos. Pure Math., Vol. 4, Amer. Math. Soc., Providence, R. I., 1961, pp. 33-49.

4. K. de Leeuw, On $L^{p}$ multipliers, Ann. of Math. (2) 81 (1965), 364-379.

5. E. Hewitt, A survey of abstract harmonic analysis. Some aspects of analysis and probability, Surveys in Applied Mathematics, Wiley, New York, 1958.

6. E. Hewitt and I. Hirschman, A maximum problem in harmonic analysis, Amer. J. Math. 76 (1954), 839-852.

7. I. I. Hirschman, Jr., On multiplier transformations, Duke Math. J. 26 (1959), 221-242.

8. L. Hörmander, Estimates for translation-invariant operators in $L^{p}$ spaces, Acta Math. 104 (1960), 93-140.

9. L. H. Loomis, An introduction to abstract harmonic analysis, Van Nostrand, New York, 1953.

10. M. Riesz, Sur les maxima des formes bilinéaires et sur les fonctionnelles linéaires, Acta Math. 49 (1926), 464-497.

11. W. Rudin, Fourier analysis on groups, Interscience, New York, 1962.

12. E. M. Stein, Interpolation of linear operators, Trans. Amer. Math. Soc. 83 (1956), 482-492.

13. M. H. Taibleson, On the theory of Lipschitz spaces of distributions on Euclidean n-space.

I. Principal properties, J. Math. Mech. 13 (1964), 407-479.

14. A. Weil, L'intégration dans les groupes topologiques et ses applications, Actualités Sci. Ind. No. 869, Hermann, Paris, 1940.

15. A. Zygmund, Trigonometric series, 2nd ed., Vols. I, II, Cambridge Univ. Press, New York, 1959.

STANFORD UNIVERSITY,

Stanford, California

THE JOHNS HOPKINS UNIVERSITY,

BALTIMORE, MARYLAND 